\title{
GESTAÇÃO NA ADOLESCÊNCIA: DESCRIÇÃO E ANÁLISE DA ASSISTÊNCIA RECEBIDA
}

\author{
Náira de Oliveira CAMINHA ${ }^{\mathrm{a}}$, Lydia Vieira FREITAS ${ }^{\mathrm{b}}$, Thaís Marques LIMAc, Linicarla Fabíole
} de Souza GOMES ${ }^{d}$, Marta Maria Soares HERCULANO ${ }^{e}$, Ana Kelve de Castro DAMASCENO ${ }^{f}$

\section{RESUMO}

Objetiva-se descrever e analisar a assistência pré-natal às adolescentes, em relação ao Programa de Humanização no Pré-natal e Nascimento (PHPN), através de um estudo descritivo, transversal e quantitativo realizado entre março e julho de 2009, a partir de formulário e entrevista com 200 adolescentes, em maternidade de referência em obstetrícia do SUS. As jovens realizaram pré-natal em serviço público (96,4\%), o iniciaram no primeiro trimestre (47,4\%), não obtiveram o mínimo de consultas (52,6\%), receberam suplemento de ferro (96,9\%), imunização antitetânica $(80,5 \%)$ e foram pouco orientadas (46,0\%). Quanto aos exames laboratoriais, foram realizados na primeira consulta (80,0\%), sendo apenas um terço deles repetidos no terceiro trimestre gestacional. Conclui-se que o PHPN não está sendo realizado integralmente, conforme o Ministério da Saúde preconiza, e a assistência pré-natal deve ser melhorada, principalmente na captação precoce, continuidade da assistência, solicitação da segunda amostra dos exames e oferta de orientações.

Descritores: Saúde do adolescente. Gravidez na adolescência. Cuidado pré-natal. Enfermagem.

\section{RESUMEN}

El objetivo es describir y analizar la atención prenatal a las adolescentes con relación al Programa de Humanización del Prenatal y Nacimiento (PHPN). Es un estudio descriptivo, transversal y cuantitativo llevado a cabo de marzo a julio de 2009 con forma y entrevista a 200 adolescentes después del parto en una maternidad de referencia. Las jóvenes recibieron atención prenatal en el servicio público (96,4\%), esta atención se inició en el primer trimestre (47,4\%), las mujeres no obtuvieron el mínimo de consultas (52,6\%), aunque recibieron suplementos de hierro (96,9\%) y vacunas contra el tétanos (80,5\%), y tuvieron pocas orientaciones (46,0\%). Los exámenes se realizaron en la primera visita (80,0\%), con una parte repetidos en el tercer trimestre. Se concluye que el PHPN no se está realizando en plena conformidad con lo que el Ministerio de Salud recomienda, y a pesar de haber sido satisfactoria en algunos puntos deberían mejorar.

Descriptores: Salud del adolescente. Embarazo en adolescencia. Atención prenatal. Enfermería.

Título: Embarazo en la adolescencia: descripción y análisis de la ayuda recibida.

\section{ABSTRACT}

This work is aimed at describing and analyzing prenatal care to teenage women through the Brazilian Prenatal and Birth Humanization Program (BPBHP). It's a descriptive quantitative study conducted between March and July 2009 based on a form and interview with 200 teenage women during the postpartum period, in a maternity ward of Sistema Único de Saúde (Brazilian Unified Health System), which is considered a reference in obstetric care. The young women received prenatal care through the public service (96.4\%) which began during the first trimester (47.4\%), they didn't have the minimum medical appointments required (52.6\%), took iron supplements (96.9\%), received tetanus immunization (80.5\%) and didn't have enough orientation (46.0\%). The laboratory tests were performed during their first medical appointment (80.0\%), but only a third were repeated in the third trimester. Therefore, the BPBHP doesn't meet all the standards set by the Ministério da Saúde (Ministry of Health), and there are improvements to be made in the early service phase, ongoing care, demand for second exam samples and availability of orientation.

Descriptors: Adolescent health. Pregnancy in adolescence. Prenatal care. Nursing.

Title: Pregnancy in adolescence: description and analysis of care provided.

a Especialista em Gestão em Saúde. Residente em Saúde da Mulher e da Criança pela Universidade Federal do Ceará (UFC). Fortaleza, Ceará (CE), Brasil.

b Doutoranda em Enfermagem pela UFC. Docente da Faculdade Metropolitana de Fortaleza (FAMETRO). Fortaleza-CE, Brasil.

c Doutoranda em Enfermagem pela UFC. Fortaleza-CE, Brasil.

d Mestranda em Enfermagem pela UFC. Enfermeira assistencial da Maternidade Escola Assis Chateaubriand (MEAC). Docente da FAMETRO. Fortaleza-CE, Brasil.

e Mestre em Enfermagem. Enfermeira obstétrica da MEAC. Docente da Faculdade Integrada da Grande Fortaleza. Fortaleza-CE, Brasil.

f Doutora em Enfermagem. Professora Adjunta IV de Enfermagem da UFC. Tutora do Programa de Educação Tutorial (PET) da UFC. Fortaleza-CE, Brasil. 


\section{INTRODUÇÃO}

A gravidez na adolescência representa um problema de saúde pública, contribuindo para aumentar as estatísticas de morbimortalidade por causas obstétricas, devido ao maior risco gestacional e neonatal.

A adolescência é permeada por um complexo processo de crescimento e desenvolvimento no qual se atinge a maturidade física e a capacidade reprodutiva, sem, contudo, atingir a maturidade psicológica e social. Adolescente é o indivíduo com idade entre 10 e $19 \operatorname{anos}^{(1)}$, o que corresponde a uma população de mais de 39 milhões de indivíduos no Brasil ${ }^{(2)}$.

As causas de morbidade dos adolescentes em 2006 no Brasil envolveram principalmente gravidez, parto e puerpério $(49,26 \%)$, respondendo por praticamente metade das causas de morbidade nesta população $o^{(3)}$. No entanto, os riscos da gravidez na adolescência não se resumem aos biológicos e obstétricos, sendo também determinados por fatores psicossociais, econômicos e sociais, aliados ao atendimento pré-natal inadequado. ${ }^{(4)}$

Considerando tais dados e indicadores, verifica-se a importância de uma atenção pré-natal de qualidade e humanizada. Este atendimento é essencial para a saúde materna e neonatal, especialmente entre adolescentes, que devem receber uma assistência pré-natal multiprofissional e interdisciplinar capaz de amparar integralmente a jovem gestante, oferecendo, além dos procedimentos básicos, cuidados especiais que possam prevenir as complicações físicas, sociais e emocionais das adolescentes grávidas(4).

Analisar a assistência pré-natal é necessário, pois esse serviço, quando de qualidade, contribui para a diminuição da morbimortalidade materna e neonatal. A avaliação é baseada nos parâmetros estabelecidos pelo Ministério da Saúde (MS), em especial os preconizados pelo Programa de Humanização no Pré-natal e Nascimento (PHPN), instituído em 2000. Este programa adota medidas para a melhoria do acesso, da cobertura e da qualidade da assistência pré-natal, por meio da análise de procedimentos mínimos a serem realizados por todas as mulheres durante o ciclo gravídico-puerperal ${ }^{(5)}$.

O PHPN preconiza que a assistência pré-natal deve ser realizada de modo a obter captação precoce da gestante; elenco mínimo de seis consultas pré-natais; escuta ativa e orientações necessárias; participação em atividades educativas; estímulo ao parto normal; anamnese e exame clínico obstétrico; solicitação de exames laboratoriais na primeira consulta e na trigésima semana gestacional; imunização anti-tetânica; avaliação e monitoramento do estado nutricional; tratamento das intercorrências da gestação; classificação do risco gestacional; atendimento a gestante com problemas e comorbidades com serviço de referência e contra-referência; registro em prontuário e cartão da gestante; além de atenção à mulher e recém-nascido $(\mathrm{RN})$ no parto e no puerpério ${ }^{(5)}$.

Enfoca-se a importância da realização das atividades preconizadas no PHPN pelos profissionais envolvidos no cuidado à gestante, dentre eles o enfermeiro, presente na assistência pré-natal de baixo e alto risco e questiona-se: Como está sendo ofertada a assistência pré-natal à adolescente? Assim, objetiva-se descrever e analisar a assistência pré-natal às adolescentes com relação ao PHPN.

\section{METODOLOGIA}

Estudo descritivo, transversal e quantitativo, realizado no Alojamento Conjunto (AC) de uma maternidade pública de referência terciária localizada em Fortaleza/CE.

A população foi composta por puérperas adolescentes atendidas no serviço de obstetrícia da instituição por ocasião do nascimento do seu filho no período de março a julho de 2009. Considerou-se a fórmula para cálculo de amostra com populações finitas, onde se considerou como população o número de adolescentes atendidas nesse serviço nos quatro meses imediatamente anteriores à coleta $(\mathrm{N}=396)$, sendo estabelecido para a definição do seu tamanho um intervalo de confiança de 95\%, um nível de significância de 0,05 e uma prevalência prevista de 50\% para os casos de adolescentes vivenciando o puerpério no AC, obtendo-se uma amostra de 196 adolescentes. Para seleção da amostra utilizamos como critérios de inclusão àquelas adolescentes que haviam realizado pelo menos uma consulta de pré-natal.

O instrumento de coleta de dados utilizado foi um formulário contendo questões sobre as características socioeconômicas, história gineco-obstétrica, informações sobre a gestação atual e o pré-natal realizado.

A coleta de dados foi realizada entre março a julho de 2009, por meio de entrevistas aplicadas às puérperas em até 48 horas pós-parto sobre a assistência pré-natal recebida; análise do cartão pré-natal e prontuário hospitalar. Os dados coletados 
foram analisados por meio do programa Statistical Package for the Social Sciences (SPSS) versão 17.0 e apresentados em tabelas, utilizando o teste exato de Fisher considerando o intervalo de confiança e nível de significância já mencionados no cálculo amostral. Com base na análise estatística, a interpretação dos resultados foi realizada à luz da literatura publicada sobre a temática.

Os aspectos éticos da pesquisa com seres humanos foram preservados, mediante a assinatura do Termo de Consentimento Livre e Esclarecido pelo responsável da adolescente e aprovação do Comitê de Ética em Pesquisa (CEP) da maternidade onde foi realizado o estudo sob protocolo número 76/08. Desta forma, foram cumpridos os preceitos éticos e legais da Resolução n. ${ }^{\circ}$ 196/96 do Conselho Nacional de Saúde - CNS.

\section{RESULTADOS E DISCUSSÕES}

Quanto às características das participantes do estudo, as adolescentes apresentaram idades entre 12 e 19 anos, sendo predominante a faixa etária de 15 a 19 anos, com 182 (91,0\%) jovens (média=16,76 anos; moda $=17$ anos); $111(55,5 \%)$ tiveram menos de oito anos de estudo e 129 (64,5 - sendo 60,0\% em união consensual e 4,5\% casadas) viviam com o pai do RN ou outro companheiro.

Agora iniciaremos a análise e discussão do foco do presente estudo: a assistência pré-natal recebida por adolescentes.

Tabela 1 - Caracterização da assistência pré-natal recebida por adolescentes. Fortaleza, CE, 2009.

\begin{tabular}{|c|c|c|}
\hline Variáveis $(n=196)$ & n & $\%$ \\
\hline \multicolumn{3}{|l|}{ Local de realização do pré-natal } \\
\hline Rede pública & 189 & 96,4 \\
\hline Rede privada & 4 & 2,1 \\
\hline Redes pública e privada & 3 & 1,5 \\
\hline \multicolumn{3}{|l|}{ Pré-natal direcionado a adolescente } \\
\hline Sim & 53 & 27,0 \\
\hline Não & 143 & 73,0 \\
\hline \multicolumn{3}{|l|}{ Realização da primeira consulta pré-natal } \\
\hline Até o $4^{\circ}$ mês & 126 & 64,3 \\
\hline A partir do $4^{\circ}$ mês & 70 & 35,7 \\
\hline \multicolumn{3}{|l|}{ Número mínimo de consultas adequadas } \\
\hline A partir de 1 consulta no $1^{\circ}$ trimestre & 91 & 46,4 \\
\hline A partir de 3 consulta no $3^{\circ}$ trimestre & 98 & 50,0 \\
\hline \multicolumn{3}{|l|}{ Número total de consultas } \\
\hline $1-5$ & 103 & 52,6 \\
\hline 6 ou mais & 91 & 46,4 \\
\hline Não respondeu & 2 & 1,0 \\
\hline \multicolumn{3}{|l|}{ Prescrição do sulfato ferroso } \\
\hline Sim & 190 & 96,9 \\
\hline Não & 5 & 2,6 \\
\hline Não respondeu & 1 & 0,5 \\
\hline \multicolumn{3}{|l|}{ Imunização antitetânica } \\
\hline Imunizadas & 161 & 82,1 \\
\hline Não imunizadas & 35 & 17,9 \\
\hline
\end{tabular}


Das jovens entrevistadas, $189(96,4 \%)$ realizaram o pré-natal na rede pública de saúde, algo esperado, considerando que a pesquisa foi realizada em uma maternidade pública de referência, onde muitas adolescentes são acompanhadas no pré-natal e para onde são encaminhadas gestantes de risco atendidas em outros serviços de saúde.

A gravidez na adolescência requer acompanhamento diferenciado, com adoção de um protocolo de risco, visitas domiciliares e inserção precoce nos programas de saúde da mulher, do adolescente e da família, que devem estar integrados para melhor atender esta clientela ${ }^{(6)}$. Ao avaliar o direcionamento da assistência para a população adolescente, constatou-se que somente $53(27,0 \%)$ receberam atendimento especializado nas características gestacionais inerentes à adolescência.

Através de cruzamento entre a variável idade e direcionamento da assistência observou-se que $52,4 \%(9 / 17)$ das adolescentes precoces (até 14 anos) foram acompanhadas em pré-natal especializado, contra $24,6 \%(44 / 179)$ das adolescentes tardias, havendo correlação estatisticamente significante ( $\mathrm{p}=0,02$ - Teste Exato de Fisher) entre direcionamento da assistência e idade das adolescentes. Tal resultado pode estar relacionado ao fato de adolescentes precoces estarem mais vulneráveis aos riscos, sendo, portanto, mais frequentemente referidas ao pré-natal especializado.

O MS preconiza que a primeira consulta pré-natal seja realizada o mais precocemente possível, até no máximo o quarto mês gestacional ${ }^{(5)}$, porém, somente $126(64,3 \%)$ puérperas referiram a primeira consulta até a $16^{\text {a }}$ semana. Este resultado impossibilita o acompanhamento adequado e integral, as intervenções e encaminhamentos oportunos na gravidez. Estudo realizado em Portugal avaliou 10.656 partos e verificou que as adolescentes tiveram pior seguimento (46,4\%) iniciando pré-natal após 120 dias gestacionais $^{(7)}$. Estudo realizado no Paraná avaliou o atendimento à criança e às mães nas unidades básicas a partir da investigação de 41 óbitos em menores de cinco anos e constatou que apenas $38,5 \%$ das mães haviam iniciado pré-natal precocemente ${ }^{(8)}$.

Outro importante aspecto observado na avaliação da qualidade do pré-natal refere-se ao número de consultas. O ideal é que sejam realizadas no mínimo seis consultas de pré-natal durante toda a gestação, sendo uma no primeiro, duas do segundo e três no último trimestre da gravidez ${ }^{(5)}$.
No entanto, observou-se que somente 91 (46,4\%) adolescentes realizaram pelo menos seis consultas, chegando uma entrevistada a realizar 13 consultas (média $=5,35 ;$ moda $=5)$ e as demais $(52,6 \%)$ não realizaram o número de consultas preconizado. Quando aos trimestres, no primeiro, (média $=0,71 ;$ moda $=0$ ) 91 (46,4\%) adolescentes alcançaram o preconizado, no segundo, (média =2,10; moda=3) 139 (70,9\%) e no terceiro, (média $=2,52$; moda $=3) 98(50,0 \%)$.

Diante do exposto, evidencia-se a necessidade de maior atenção dos serviços de saúde à continuidade da assistência. Em 2002 aproximadamente $14 \%$ e $27 \%$ das gestantes da região Nordeste e Sul, respectivamente, realizaram seis consultas de pré-natal ${ }^{(9)}$, no entanto, passados sete anos, tal indicador básico ainda não apresentou frequência superior a 46,4\% entre as adolescentes, população exposta a fatores de riscos adicionais e que necessitam de uma atenção mais regular.

A prescrição de sulfato ferroso apresentou-se satisfatória, abrangendo 190 (96,9\%) gestantes. Resultado importante, pois a suplementação de ferro é indispensável para a prevenção/tratamento da anemia fisiológica e/ou ferropriva na gestação, e repõe as reservas de ferro materno, preparando a mulher para as perdas sanguíneas do parto e puerpério.

A vacinação antitetânica foi avaliada e é importante para a prevenção do tétano materno e neonatal, sendo necessária a aplicação da dose imunizante da vacina antitetânica durante o pré-natal ${ }^{(5)}$. A partir da segunda dose do esquema o RN é considerado imunizado, e somente com a terceira dose imuniza-se mãe e RN, devendo ser ainda administrada uma dose de reforço para aquelas já imunizadas com a última dose há mais de 5 anos.

Neste estudo, 161 (82,1\%) puérperas receberam a imunização necessária durante o pré-natal. Das entrevistadas que referiram somente uma dose ou nenhuma e não receberam vacinação anterior, somando àquelas que não realizaram o pré-natal obtivemos um total de $35(17,9 \%)$ mulheres que estavam, junto com seus RN, susceptíveis ao tétano. Corroborando com tais resultados, no Rio Grande do Sul, aproximadamente $80 \%$ das puérperas receberam imunização antitetânica durante o pré-natal ${ }^{(10)}$.

O MS preconiza a realização de uma rotina mínima de exames laboratoriais, com solicitação na primeira consulta dos exames laboratoriais: Tipagem de sangue, Hematimetria, Veneral Disease Research Laboratory (VDRL), Sumário de Urina 
Tabela 2 - Exames realizados por adolescentes na assistência pré-natal. Fortaleza, CE, 2009.

\begin{tabular}{lcccc}
\hline \multicolumn{1}{r}{ Variáveis $(\mathbf{n = 1 9 6})$} & \multicolumn{2}{c}{ Primeira consulta } & \multicolumn{2}{c}{ Consulta após a $\mathbf{3 0}^{\mathbf{a}}$ semana } \\
& $\mathbf{n}$ & $\mathbf{\%}$ & $\mathbf{n}$ & $\mathbf{\%}$ \\
\hline Tipagem sanguínea & 184 & 93,8 & - & - \\
Hematimetria & 164 & 83,7 & - & - \\
VDRL & 166 & 84,7 & 63 & 32,5 \\
Sumário de Urina & 178 & 90,8 & 80 & 41,2 \\
Glicemia de Jejum & 162 & 82,7 & 50 & 25,8 \\
Sorologia para HIV & 163 & 83,2 & 47 & 24,2 \\
Sorologia para Toxoplasmose & 63 & 32,1 & - & - \\
Sorologia para Hepatite B & - & - & 46 & 23,7 \\
\hline
\end{tabular}

Fonte: Cartão pré-patal e/ou Prontuário institucional.

(URINA tipo I), Glicemia de Jejum, sorologia para HIV 1 e 2. Os quatro últimos testes dever ser repetidos próximo à trigésima semana, juntamente com a sorologia para a Hepatite B. As sorologias para toxoplasmose, rubéola e citomegalovírus devem ser incluídas sempre que disponível ${ }^{(11)}$.

Verificou-se que mais de $80 \%$ das puérperas avaliadas realizaram a maioria dos exames preconizados para a primeira consulta, exceto a sorologia para toxoplasmose, que foi realizada somente por 63 $(32,1 \%)$ delas. Ao comparar a situação de realização de seis exames laboratoriais e da sorologia para HIV em 2002, percebe-se a melhoria ao considerar que no Nordeste os índices eram de 12\% e os melhores indicadores no período, pertencentes ao Sul e Sudeste, não alcançaram $60 \%$ de cobertura ${ }^{(9)}$.

Percebem-se melhorias na realização desta rotina mínima ao longo dos anos, no entanto, a solicitação e registro de tais exames merece, ainda, uma atenção dos profissionais, como também dos laboratórios, em disponibilizarem os resultados em tempo hábil. Deve-se também ponderar que $20 \%$ das gestantes não realizaram todos os exames preconizados, fato preocupante, considerando que estes são exames básicos e imprescindíveis para o acompanhamento ideal das gestantes.

A sorologia para toxoplasmose, realizada por somente $63(32,1 \%)$ das entrevistadas, é incluído quando disponível. Questiona-se, porém, se a não realização do exame deu-se por indisponibilidade ou por falta de solicitação profissional. Considerando as repercussões desta infecção na gestação sobre a saúde fetal, percebe-se a importância da detecção precoce da mesma, a fim de reduzir as co-morbidades associadas, além de detectar as mulheres suscetíveis e reforçar as orientações necessárias.

A repetição da rotina laboratorial básica na trigésima semana é importante frente à possibilidade da "janela imunológica", e contaminação da gestante pelo HIV, sífilis, hepatite B, além da possibilidade de se desenvolver diabetes e hipertensão gestacional, ou infecção urinária, patologias que envolvem risco adicional para a mulher e recém-nascido.

Apesar da visível importância, próximo à trigésima semana, a realização de exames foi pouco frequente, sendo mais requisitado o sumário de urina, que não atingiu 50,0\% de realização dentre as adolescentes. Percebe-se daí que pouco mais de um terço das adolescentes que realizaram os exames na primeira consulta, os repetiram na segunda, algo inquietante, considerando que ambos são importantes para o acompanhamento pré-natal e os últimos exames são mais fidedignos para o momento do parto.

Estudo que avaliou a atenção pré-natal de gestantes a termo usuárias do Sistema Único de Saúde - SUS em Juíz de Fora/MG constatou que no ano de 2004, 94,3\% das gestantes realizaram uma tipagem sanguínea durante o pré-natal e os demais exames (Hematimetria; VDRL; Glicemia e Urina tipo I) variaram de $56,1 \%$ a $66,1 \%$ com apenas um registro e em média 29\% tinham um segundo registros destes exames no seu cartão pré-natal ${ }^{(12)}$. O sumário de urina, importante para detectar fatores complicadores como a infecção do trato urinário (ITU), foi repetido por 80 $(41,2 \%)$ gestantes, algo preocupante considerando que a ITU é a complicação obstétrica mais freqüente. 
Tabela 3 - Procedimentos realizados na assistência pré-natal com adolescentes. Fortaleza, CE, 2009.

\begin{tabular}{lcc}
\hline \multicolumn{1}{c}{ Variáveis (n=196) } & n & \% \\
\hline Cálculo da Idade Gestacional & 178 & 90,8 \\
Avaliação Ponderal & 171 & 87,2 \\
Verificação da Pressão Arterial (PA) & 187 & 95,4 \\
Medição de Altura Uterina (AU) & 183 & 93,4 \\
Palpação Obstétrica & 164 & 83,7 \\
Avaliação de Edema & 101 & 51,5 \\
Ausculta de Batimentos Cárdio-Fetais (BCF) & 185 & 94,4 \\
Avaliação dos Movimentos Fetais & 174 & 88,8 \\
\hline
\end{tabular}

Fonte: Cartão pré-natal e/ou Prontuário institucional.

Com relação ao cálculo da Idade Gestacional (IG), $178(90,8 \%)$ adolescentes afirmaram ter sido informada sobre a sua IG e Data Provável do Parto (DPP), e essas informações foram registradas no cartão da gestante.

A avaliação ponderal, que avalia a existência de risco nutricional e o ganho de peso materno, foi referida por $171(87,2 \%)$ entrevistadas. Em Recife/ $\mathrm{PE}$, estudo revelou que somente $31 \%$ das mulheres referiram este procedimento em todas as consultas de pré-natal ${ }^{(13)}$. A avaliação ponderal propicia a detecção de possíveis patologias maternas que podem interferir negativamente no desenvolvimento fetal, como também no trabalho de parto, portanto, é relevante para todas as gestantes. Existem ferramentas práticas e acessíveis, como o cálculo do Índice de Massa Corporal (IMC), o que torna a não avaliação injustificável.

A aferição da PA, fundamental para a detecção precoce de possíveis formas clínicas da Síndrome Hipertensiva Gestacional (SHG), foi relatada por $187(95,4 \%)$ puérperas.

A medição da AU foi referida por 183 (93,4\%) jovens entrevistadas. Essa medição estima o crescimento fetal e através do traçado gerado no gráfico ao longo do acompanhamento pré-natal é possível detectar alterações fetais como restrição do crescimento intrauterino. Em Pelotas/RS, a aferição da AU e da PA alcançaram respectivamente $96,4 \%$ e $99,0 \%{ }^{(10)}$.

Diante destas referências, percebe-se que a aferição da AU e da PA estão sendo realizadas de forma satisfatória na assistência pré-natal das jovens puérperas de nosso estudo. Porém quando se tem a SHG como importante causa de morbimortalidade materna, havendo nos ex tremos de idade incremento do risco, a cobertura de $100 \%$ das gestantes em acompanhamento pré-natal com monitorização de pressão arterial mensal se torna imprescindível no combate a esse indicador.

A palpação obstétrica para identificar a situação, apresentação fetal e altura da apresentação, foi referida por $164(83,7 \%)$ adolescentes. Esta porcentagem, apesar de alta, é preocupante, pois a palpação obstétrica é um dos pontos mais básicos a ser realizado durante o exame físico no pré-natal. Em Recife/PE, este procedimento foi mencionado por $42,1 \%$ das puérperas estudadas, como havendo sido realizado no mínimo duas vezes ${ }^{(13)}$.

A avaliação de edema, que pode ser fisiológico ou patológico, foi citada somente por 101 $(51,5 \%)$ entrevistadas. É importante ressaltar que tal procedimento pode ser realizado sutilmente e a gestante pode não percebê-lo. Pesquisa realizada com puérperas em duas maternidades de referência de Pernambuco obteve resultados inferiores aos encontrados no presente estudo, com percentual de detecção de edema igual a $16,4^{\%} \%^{(1))}$.

A ausculta dos BCF e a avaliação dos movimentos fetais são fundamentais para observar a vitalidade do feto, sendo aquele procedimento relatado por 185 $(94,4 \%)$ adolescentes e este por $174(88,8 \%)$. Em Pelotas, $97,8 \%$ das mulheres referiu ter sido realizada a ausculta do BCF durante o pré-natal ${ }^{(10)}$, resultado semelhante ao encontrado neste estudo. Através da monitorização fetal, o pré-natalista pode detectar e intervir precocemente em sinais de sofrimento fetal, prevenindo óbitos fetais evitáveis.

Observamos em relação às orientações durante o pré-natal que $166(84,7 \%)$ mulheres receberam alguma orientação durante o ciclo gravídico puerperal. Sabe-se que a realização de ações educativas é imprescindível 
Tabela 4-Distribuição das orientações recebidas durante o pré-natal de puérperas adolescentes atendidas na MEAC no período de março a julho. Fortaleza, Ceará, Brasil, 2009.

\begin{tabular}{lcc}
\hline \multicolumn{1}{c}{ Variáveis $(\mathbf{n = 1 9 6})$} & $\mathbf{n}$ & \% \\
\hline À̀ assistência pré-natal & 110 & 56,1 \\
Aos sintomas de gravidez & 108 & 55,1 \\
Às modificações corporais e emocionais na gestação & 84 & 42,9 \\
Ao parto & 89 & 45,4 \\
Aos cuidados puerperais & 84 & 42,9 \\
À puericultura & 75 & 38,2 \\
Aos cuidados com o recém-nascido & 92 & 46,9 \\
À triagem neonatal & 79 & 40,3 \\
Ao aleitamento materno & 119 & 60,7 \\
Nenhuma orientação & 30 & 15,3 \\
\hline
\end{tabular}

Fonte: Adolescentes entrevistadas.

em todas as etapas deste ciclo, mas é no pré-natal que a mulher deve ser mais bem orientada para que possa viver a gestação e o parto de forma positiva, com mais saúde e menos risco de complicações ${ }^{(14)}$.

As menores frequências encontradas foram quanto às orientações sobre a consulta de puericultura e a triagem neonatal, que referem-se à saúde do neonato. No Rio Grande do Sul, estudo que avaliou crianças de 12 a 16 meses quanto ao acompanhamento na puericultura observou que $53,2 \%$ das crianças não compareceram regularmente às consultas e 66,2\% das mães ou responsáveis não consideravam a consulta necessária ${ }^{(15)}$. Assim, reforçamos a importância de práticas educativas que discutam à relevância da puericultura para avaliação do crescimento, desenvolvimento e saúde da criança, além da sensibilização de profissionais pelos gestores quanto à necessidade de oferecer tais informações através de campanhas, atividades educativas individuais e coletivas.

As questões referentes ao aleitamento materno foram orientadas com mais frequência, sendo ofertadas a 119 (60,7\%) jovens. Algumas mulheres, entretanto, receberam informações educativas no pós-parto, e não durante a assistência pré-natal. Observou-se em estudo desenvolvido com 20 puérperas em Ribeirão Preto que o conhecimento das mães sobre a amamentação é um dos fatores que contribuem para a adoção desta prática e o tempo de permanência do binômio nas unidades de puerpério podem não ser suficientes para a obtenção das orientações necessárias para a prática adequada da amamentação $^{(16)}$. Diante disso, os profissionais que realizam o pré-natal devem orientar $100 \%$ das mulheres durante as consultas, e reforçar a necessidade da consulta de puericultura, também fundamental para que as mães percebam a importância da amamentação e adotem essa prática de forma eficaz.

\section{CONCLUSÕES}

Conclui-se que apesar de alguns pontos preconizados pelo PHPN estarem sendo desenvolvido de forma satisfatória, o acompanhamento pré-natal precisa melhorar na captação precoce da gestante (64,3\%) e na busca na continuidade da assistência (46,4\%), visando aumentar a assiduidade das mesmas às consultas.

Ao analisar os indicadores mínimos exigidos pelo PHPN, observa-se que itens como: a realização da primeira amostra dos exames laboratoriais (80,0\%), a imunização antitetânica (82,1\%), suplementação férrica $(96,9 \%)$ e avaliação ponderal (87,2\%) encontram-se no percentual satisfatório, porém não ideal.

Houve baixo percentual em aspectos essenciais, como realização do mínimo de consultas $(46,4 \%)$, a coleta da segunda amostra dos exames laboratoriais $(<41,3 \%)$, bem como as orientações em geral $(<60,8 \%)$, onde se podem analisar as ações de educação em saúde realizadas de maneira precária e não prioritárias pelos profissionais de saúde, dentre eles o enfermeiro.

É importante ressaltar que os resultados aqui encontrados evidenciam o perfil da assistência pré-natal de adolescentes atendidas no serviço de obstetrícia da maternidade onde ocorreu o estudo, 
não devendo ser generalizada para a população geral de adolescentes do município, estado ou país.

Estes resultados contribuem para evidenciar a assistência prestada às gestantes adolescentes, servindo de subsídio para elaboração de estratégias, correção de falhas, e estímulo a estudos que reflitam o perfil geral da assistência pré-natal à população adolescente. Por fim, sugere-se a inclusão da consulta puerperal e definição do profissional responsável pelo atendimento na realização de estudos futuros.

\section{REFERÊNCIAS}

1 World Health Organization. Adolescent hearth [Internet] 2008 [cited 2008 sep 11] Available from: http://www.who.int/topics/adolescent_health/en/

2 Ministério da Saúde (BR). DATASUS/IBGE: população residente - projeções intercensitárias [Internet]. Brasília (DF); 2009 [citado 2010 jan 31]. Disponível em: http://tabnet.datasus.gov.br/cgi// tabcgi.exe?ibge/cnv/popuf.def.

3 Ministério da Saúde (BR). Databases. Indicadores e Dados Básico - Brasil 2008. IDB-2008 [Internet]. Brasília (DF);2008 [citado 2009 dez 11]. Disponível em: http:// tabnet.datasus.gov.br/cgi/idb2008/matriz.htm

4. Sant'Anna MJC, Coates V. Gravidez na adolescência: um novo olhar. In: Secretaria de Saúde (São Paulo, SP). Manual de atenção à saúde do adolescente. São Paulo: SMS; 2006. P. 153-8.

5 Ministério da Saúde (BR). Programa Humanização do Parto: humanização no pré-natal e nascimento. Brasília (DF): Ministério da Saúde; 2002.

6 Souza ML, Burgardt D, Ferreira LAP, Bub MBC, Monticelli M, Lentz HE. Meninas catarinas: a vida perdida ao ser mãe. Rev Esc Enferm USP. 2010;44(2):318-23.

7 Metello J, Torgal M, Viana R, Martins L, Maia M, Casal E, et al . Desfecho da gravidez nas jovens adolescentes. Rev Bras Ginecol Obstet. 2008;30(12):620-5

\footnotetext{
Endereço da autora / Dirección del autor / Author's address

Náira de Oliveira Caminha

Rua Professor Odílio Filho, 253A, Passaré

60743-750, Fortaleza, CE

E-mail:nairacaminha@yahoo.com.br
}

8 Jodas DA, Scochi MJ, Moura MB, Tiwata MZ. Atendimento à criança e às mães: investigação do óbito evitável em menores de cinco anos. Rev Gaúcha Enferm. 2011;32(4): 669-675.

9 Ministério da Saúde (BR). Avaliação nacional do Programa de Humanização do Pré-natal e Nascimento. Rev Saude Publica. 2008;42(2): 383-387.

10 Rasia ICRB, Albernaz E. Atenção pré-natal na cidade de Pelotas, Rio Grande do Sul, Brasil. Rev Bras Saude Mater Infant. 2008;8(4):401-10.

11 Ministério da Saúde (BR). Secretaria de Atenção à Saúde. Departamento de Ações Programáticas Estratégicas. Pré-natal e puerpério: atenção qualificada e humanizada. Manual Técnico. $3^{\mathrm{a}}$ ed. Rev. Brasília (DF): Ministério da Saúde; 2006.

12 Coutinho T, Monteiro MFG, Sayd JD, Teixeira MTB, Coutinho CM, Coutinho LM. Monitoramento do processo de assistência pré-natal entre as usuárias do Sistema Único de Saúde em município do Sudeste brasileiro. Rev Bras Ginecol Obstet 2010;32(11):563-569.

13 Carvalho VCP, Araujo TVB. Adequação da assistência pré-natal em gestantes atendidas em dois hospitais de referência para gravidez de alto risco do Sistema Único de Saúde, na cidade de Recife, Estado de Pernambuco. Rev Bras Saude Mater Infant. 2007;7(3):309-17.

14 Rios CTF, Vieira NFC. Ações educativas no pré-natal: reflexão sobre a consulta de enfermagem como um espaço para educação em saúde. Ci Saúde Colet. 2007;12(2):477-86.

15 Vitolo MR, Gama CM, Campagnolo PDB. Frequência de utilização do serviço público de puericultura e fatores associados. J Pediatr. 2010;86(1):80-4.

16 Nakano MAS, Reis MCG, Pereira MJB, Gomes FA. O espaço social das mulheres e a referencia para o cuidado na prática da amamentação. Rev Latino-Am Enferm. 2007;15(2):230-8.

Recebido em: 03.05.2011

Aprovado em: 31.08 .2012 